Cite this: RSC Adv., 2014, 4, 20632

Received 1st March 2014 Accepted 16th April 2014

DOI: $10.1039 / c 4 r a 02941 k$

www.rsc.org/advances

\section{A Ru-catalyzed one-pot synthesis of homopropargylic amines from alkyl azides under photolytic conditions $\uparrow$}

\author{
Wook Jeong, Ji Hyung Lee, Jungjoon Kim, Won Jong Lee, Ju-Hwa Seong, \\ Jaiwook Park* and Young Ho Rhee*
}

A new synthetic method for homopropargylic amines from alkyl azides is presented. A salient feature of this reaction is the involvement of $N$ unsubstituted imines as the key intermediates, which are generated from alkyl azides by Ru catalysis under photolytic conditions. Notably, this method avoids the use of a protective group strategy in the homopropargylic amine synthesis.

Homopropargylic amines are synthetically useful building blocks that are used as versatile intermediates in numerous natural product syntheses. ${ }^{1}$ Thus, the development of efficient synthetic methods for the homopropargylic amines continues to be an important goal in synthetic organic chemistry. The most common protocol involves the addition of allenylmetal species to suitably protected imine derivatives. ${ }^{2}$ In fact, imines masked with aryl, ${ }^{3}$ alkyl, ${ }^{4}$ sulfinyl, ${ }^{5}$ or trialkylsilyl group have been frequently used for the synthesis of homopropargylic amines. ${ }^{6}$ This common approach, however, has inherent limitation because the protective groups should be removed for further functional group transformations. ${ }^{7}$ In this regard, an ideal approach would be to use the $N$-unsubstituted imines. This conceptually new approach has remained elusive because of the instability of the $N$-unsubstituted imines (Scheme 1).

Most recently, we reported that $N$-unsubstituted imines could be generated from various alkyl azides by the use of diruthenium catalyst 1 under fluorescent light conditions. ${ }^{\mathbf{8} 9}$ We also discovered that homoallylic amines could be generated in a one-pot manner by combining this catalytic imine generation with allylic boronate esters. Based upon this report, we anticipated that the treatment of alkyl azides with allenylmetal species in the presence of diruthenium catalyst could provide primary homopropargylic amines with no need of protective groups. It should be noted that $N$-unsubstituted imines could

Department of Chemistry, POSTECH (Pohang University of Science and Technology), Pohang, Kyungbuk 790-784, Korea. E-mail: pjw@postech.ac.kr; yhrhee@postech.ac. kr; Fax: +82-54-279-3399; Tel: +82-54-279-2121

† Electronic supplementary information (ESI) available. See DOI: $10.1039 / \mathrm{c} 4 \mathrm{ra02941k}$ be alternatively prepared from carbonyl precursors ${ }^{\mathbf{1 0 a}}$ by the condensation with excess basic ammonia or from alkyl cyanides by the in situ reaction with strong reducing agents. ${ }^{10 b}$ However, synthesis of homopropargylic amines relying on this process remains unknown, to our best knowledge. ${ }^{\mathbf{1 1}}$

Due to the labile nature of the $N$-unsubstituted imines, careful selection of the allenylmetal species would be a key factor for the desired one-pot transformation. This concern led us to consider the use of allenyl boronate species (Scheme 2). Under this condition, the initial formation of the imine-boronate adduct $\mathbf{A}$ (step $\mathrm{A}$ ) and the subsequent intramolecular

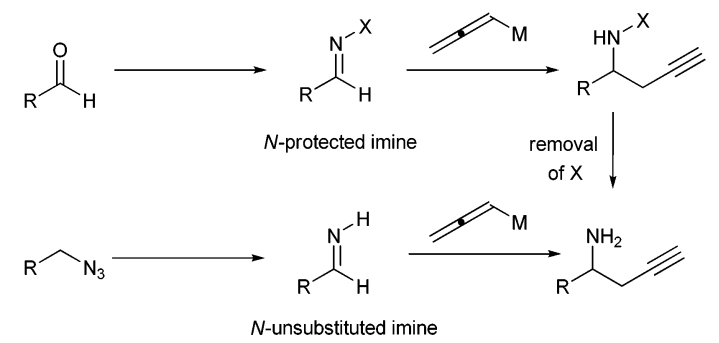

Scheme 1 Ru-catalyzed synthesis of homopropargylic amines via $N$-unsubstituted imines.

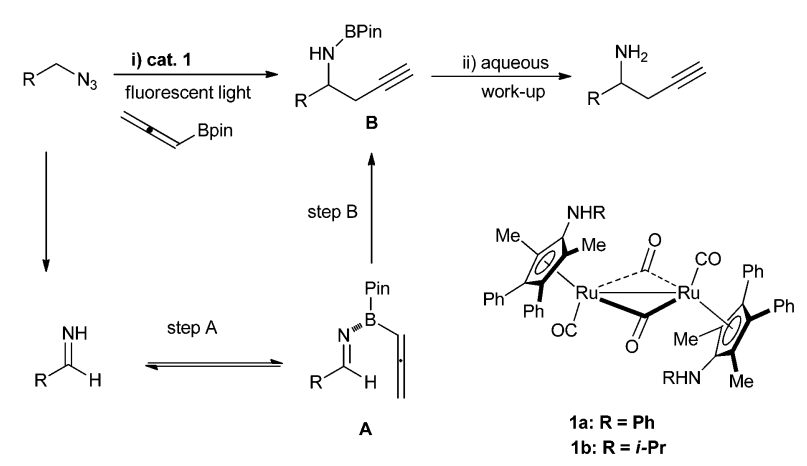

Scheme 2 Basic scheme for the one-pot synthesis of homopropargylic amines from alkyl azides. 
transfer of the allenyl moiety will generate the amine-boronate adduct B (step B), which will produce homopropargylic amines after aqueous work-up.

We initially optimized the reaction condition using benzyl azide 2 as the substrate(Table 1). Based upon our previous study, ${ }^{8}$ one-pot synthesis of homopropargylic amine was performed in the presence of $\mathrm{Ru}$ catalyst $\mathbf{1 a}(2 \mathrm{~mol} \%)$ and commercially available 3 ( 3 equiv.) in THF under illumination with $30 \mathrm{~W}$ household fluorescent light. When the reaction was performed at room temperature, the homopropargylic amine $4 a$ was obtained in 59\% NMR yield after the aqueous work-up (entry 1). ${ }^{\mathbf{1 2}}$ Notably, the yield of $\mathbf{4 a}$ was significantly lower than that for the corresponding allylic amine synthesis obtained in our previous study. ${ }^{8}$ Careful analysis of the crude reaction mixture indicated formation of significant amount of by-products such as imine trimer $\mathbf{4 b}$ in $41 \%$ yield. Using catalyst $\mathbf{1 b}$ significantly slowed the reaction (entry 2). In the light of this result, we hypothesized that the low yield of the homopropargylic amine 4 a could be explained by less favourable formation of the imine-boronate adduct $\mathbf{A}$ (step A) or the slow addition of allenyl moiety to the imine (step B). After extensive efforts, we discovered that increasing the reaction temperature to $40{ }^{\circ} \mathrm{C}$ significantly improved the yield of desired amine $4 \mathbf{a}$ up to $83 \%$ (entry 3 ). Using lower catalyst loading ( $1 \mathrm{~mol} \%$ ) at this temperature still maintained the catalytic activity with only slight decrease in the yield of $4 a$ (entry 4). Increasing the amount of boronate ester 3 to 5 equiv. somewhat improved the yield of $\mathbf{4 a}$ (entry 5). The concentration also had a notable effect on the yield of the desired product. While concentration of the solution significantly decreased the yield of $4 \mathbf{a}$ (entry 6 ), dilution showed adverse effect, increasing the yield of $4 a$ to $88 \%$

Table 1 Optimization

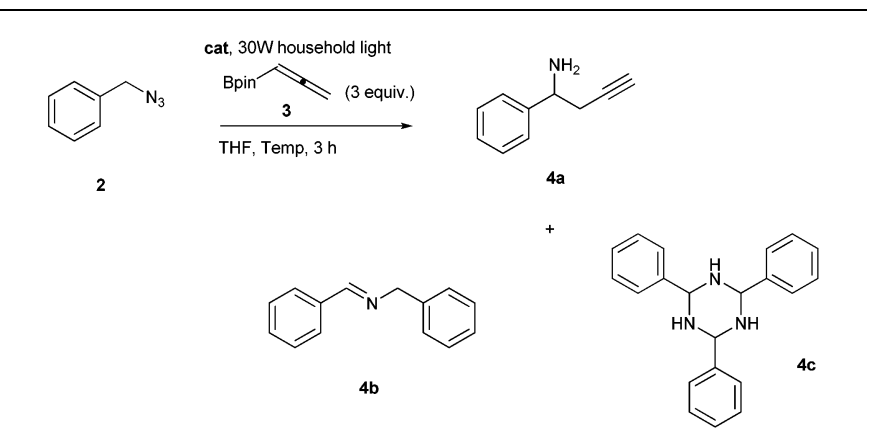

\begin{tabular}{llllll}
\hline Entry & Cat. (mol\%) & Conc. (M) & Temp. $\left({ }^{\circ} \mathrm{C}\right)$ & $\mathbf{4 a}^{a}(\%)$ & $\mathbf{4 b}^{a}(\%)$ \\
\hline 1 & 1a (2) & 0.5 & $\mathrm{rt}$ & 59 & 41 \\
$2^{b}$ & $\mathbf{1 b}(2)$ & 0.5 & $\mathrm{rt}$ & 24 & 15 \\
3 & 1a (2) & 0.5 & 40 & 83 & 17 \\
4 & 1a (1) & 0.5 & 40 & 79 & 21 \\
$5^{c}$ & 1a (1) & 0.5 & 40 & 83 & 17 \\
6 & 1a (1) & 1.0 & 40 & 71 & 29 \\
7 & 1a (1) & 0.25 & 40 & 88 & 12 \\
8 & 1a (1) & 0.25 & 50 & $94\left(81^{d}\right)$ & $<5$
\end{tabular}

${ }^{a}$ NMR yield. ${ }^{b} \sim 60 \%$ of starting material was recovered. ${ }^{c} 5$ equiv. of boronate ester 3 was used. ${ }^{d}$ Isolated yield. (entry 7). Under the optimal condition performed at $50{ }^{\circ} \mathrm{C}$ in THF $(0.25 \mathrm{M})$ in the presence of Ru catalyst $1 \mathrm{a}$ (1 mol\%), homopropargylic amine 4a was obtained in 94\% NMR yield ( $81 \%$ isolated yield) with minimal formation of the by-products $(<5 \%$, entry 8$)$.

With the optimized reaction condition for the one-pot procedure in hand, we then tested an array of alkyl azides for the desired one-pot transformation (Table 2). In the case of benzylic azides 5-8, variation of substituents at the para position of the phenyl ring showed noticeable effect (entries 1-4). For example, installing fluorine group significantly slowed the reaction (entry 4). Nevertheless, the yield of the reaction did not significantly vary. As shown in entry 5 , the scope of the reaction was successfully expanded to the synthesis of aliphatic homopropargylic amine 19. The $\alpha$-amino ester 20 possessing homopropargyl group was also produced in $61 \%$ yield (entry 6 ). Thus, the current reaction is compatible with the easily reducible ester group. Notably, homopropargylic amines bearing $\alpha$-siloxy group (entry 7 ) and $\beta$-siloxy group (entry 8 ) were also obtained in good yield to give synthetically useful 1,2- and 1,3-aminoalcohols. These examples illustrate that the potentially base-labile siloxyimine intermediates are stable under the mild reaction condition, and address the chemoselectivity of the current methodology. In

Table 2 Scope of the reaction

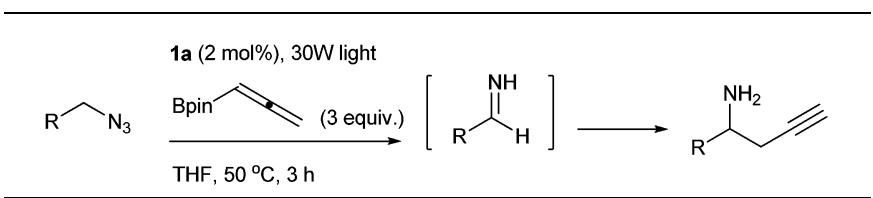

\begin{tabular}{lll}
\hline Entry $^{a}$ Reactant & Time (h) Product & Yield $^{b}(\%)$
\end{tabular}

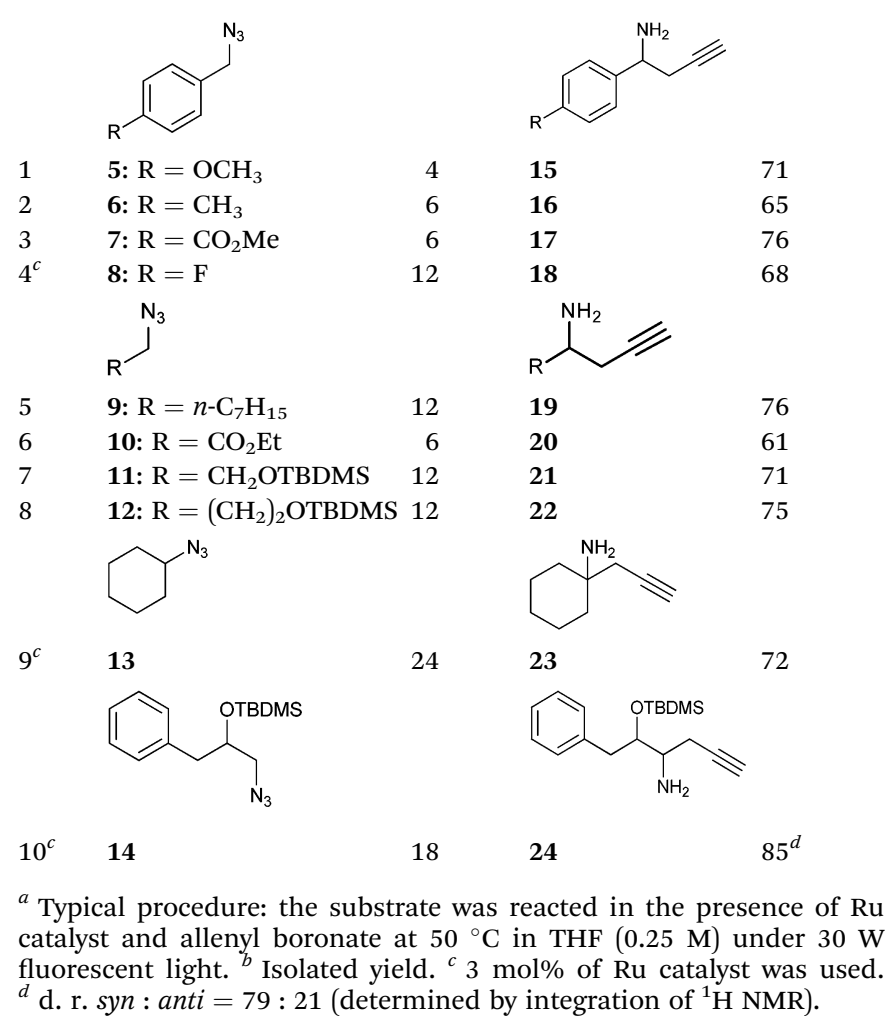




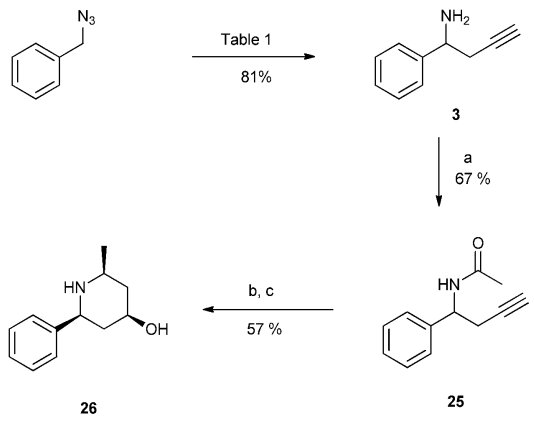

Scheme 3 A protective group-free synthesis of piperidin-4-ol from benzylic azide. ${ }^{a} \mathrm{CH}_{3} \mathrm{COCl}$ (1.2 equiv.), $\mathrm{Et}_{3} \mathrm{~N}$ (2 equiv.), $\mathrm{CH}_{2} \mathrm{Cl}_{2}, \mathrm{rt}, 5$ h. ${ }^{\mathrm{b}}$ $\left[\mathrm{Au}\left(\mathrm{PPh}_{3}\right)\right]^{+} \mathrm{NTf}_{2}{ }^{-}(5 \mathrm{~mol} \%), \mathrm{MsOH}$ (1.2 equiv.), $4 \AA$ M.S., rt, $20 \mathrm{~min}$; catecholborane (6 equiv.), rt, $10 \mathrm{~h}$.

addition to the primary alkyl azide substrates shown above, secondary cyclohexyl azide $\mathbf{1 3}$ proved to be efficient substrate even though the reaction was somewhat slower than the aliphatic primary alkyl azides. When the reaction was performed in the presence of $3 \mathrm{~mol} \%$ catalyst for $24 \mathrm{~h}$, the product 23 was obtained in $72 \%$ yield (entry 9 ). Thus, tertiary carbinylamines can be also accessed by this method. Finally, homopropargylic amine 24 was afforded in $85 \%$ yield with $\sim 4: 1$ syn-stereoselectivity. ${ }^{13}$

As discussed above, the homopropargylic amines have been used as versatile intermediates in synthetic organic chemistry(Scheme 3 ). For example, the product 24 could be used as important precursors for the synthesis of various aminodeoxysugars via metal-catalyzed/mediated cycloisomerization reaction. ${ }^{\mathbf{1 4}}$ In addition, the homopropargylic amines generated via the proposed method can be merged with other metalcatalyzed processes to access more structurally and stereochemically diverse nitrogen heterocycles in a highly efficient manner. ${ }^{15,16}$ As an example, we explored the synthesis of 2,6dialkyl-4-hydroxy-piperidine 26 by combining the current protocol with the gold(I)-catalyzed in situ cycloisomerizationreduction protocol developed by Zhang. ${ }^{15}$ While the synthesis of acetylated homopropargylic amine precursor 25 in Zhang's study requires the use of tert-butylsulfinyl group for the installation of the allenyl group, the current protocol can eliminate these additional steps. Thus, the current synthesis significantly improved the step efficiency of this catalytic reaction.

In summary, we have developed a new and efficient one-pot synthesis of homopropargylic amines from various alkyl azides. This result significantly expands the utility of $N$-unsubstituted imines in amine synthesis. Variation of allenylmetal species as well as the asymmetric version of the proposed homopropargylic amine synthesis is now under progress in our laboratory, and the result will be reported in due course.

\section{Acknowledgements}

This work was supported by the National Research Foundation funded by the Korean government (NRF-2013R1A2A2A01068684 and NRF-2012-007235).

\section{Notes and references}

1 (a) F. Alonso, I. P. Beletskay and M. Yus, Chem. Rev., 2004, 104, 3079; (b) B. E. Maryanoff, H.-C. Zhang, J. H. Cohen, I. J. Turchi and C. A. Maryanoff, Chem. Rev., 2004, 104, 1431; (c) E. M. Vieira, F. Haeffner, M. L. Snapper and A. H. Hoveyda, Angew. Chem., Int. Ed., 2012, 51, 6618; (d) T. J. A. Graham and A. G. Doyle, Org. Lett., 2012, 14, 1616; (e) B. Wu, C. Jia, X. Wang, S. Li, X. Huang and X.-J. Yang, Org. Lett., 2012, 14, 684; $(f)$ T. J. A. Graham, J. D. Shields and A. G. Doyle, Chem. Sci., 2011, 2, 980.

2 For a review on the synthesis of homopropargylic amines, see: (a) R. Bloch, Chem. Rev., 1998, 98, 1407; (b) C.-H. Ding and X.-L. Hou, Chem. Rev., 2011, 111, 1914. For selected recent examples on the synthesis of homopropargylic amines, see: (c) H. M. Wisniewska and E. R. Jarvo, Chem. Sci., 2011, 2, 807; (d) D. R. Fandrick, C. S. Johnson, K. R. Fandrick, J. T. Reeves, J. Tan, H. Lee, J. J. Song, N. K. Yee and C. H. Senanayake, Org. Lett., 2010, 12, 748.

3 For some selected papers: (a) Y. Yamamoto, W. Ito and K. Maruyama, J. Chem. Soc., Chem. Commun., 1984, 1004; (b) A. Yanagisawa, T. Suzuki, T. Koide, S. Okitsu and T. Arai, Chem.- Asian J., 2008, 3, 1793; (c) C. Wei and C.-J. Li, J. Am. Chem. Soc., 2002, 124, 5638.

4 For some selected papers: (a) H. C. Brown, U. R. Khire, G. Narla and U. S. Racherla, J. Org. Chem., 1995, 60, 544; (b) S. S. Nikam and K. K. Wang, J. Org. Chem., 1985, 50, 2193. 5 For some selected papers: (a) M. T. Robak, M. A. Herbage and J. A. Ellman, Chem. Rev., 2010, 110, 3600; (b) F. Chemla and F. Ferreira, Synlett, 2006, 2613; (c) A. Voituriez, F. Ferreira, A. Pérez-Luna and F. Chemla, Org. Lett., 2007, 9, 4705; (d) A. Voituirez, F. Ferreira and F. Chemla, J. Org. Chem., 2007, $72,5358$.

6 (a) A. Z. Gonzalez and J. A. Soderquist, Org. Lett., 2007, 9, 1081; (b) L. Leboutet, G. Courtois and L. Miginiac, J. Organomet. Chem., 1991, 420, 155.

7 (a) M. Yus, J. Gonzalez-Gomez and F. Foubelo, Chem. Rev., 2011, 111, 7774; (b) P.-O. Delaye, J.-L. Vasse and J. Szymoniak, Org. Lett., 2012, 14, 3004; (c) M. Fujita, T. Nagano, U. Schneider, T. Hamada, C. Ogawa and S. Kobayashi, J. Am. Chem. Soc., 2008, 130, 2914.

8 J. H. Lee, S. Gupta, W. Jeong, Y. H. Rhee and J. Park, Angew. Chem., Int. Ed., 2012, 51, 10851.

9 (a) Y. Do, J. Han, Y. H. Rhee and J. Park, Adv. Synth. Catal., 2011, 353, 3363; (b) Y. Do, I.-C. Hwang, M.-J. Kim and J. Park, J. Org. Chem., 2010, 75, 5740.

10 (a) M. Sugiura, K. Hirano and S. Kobayashi, J. Am. Chem. Soc., 2004, 126, 7182; (b) B. Dhudshia, J. Tiburcio and A. N. Thadani, Chem. Commun., 2005, 5551.

11 We investigated the homopropargylic amine synthesis mediated by the condensation of benzaldehyde with ammonia. Using liquid ammonia or aqueous ammonia at low temperature (below rt) with allenylboronate 3a following the protocol described in ref. $10 a$ showed poor conversion, generating the product $4 \mathrm{a}$ in $\sim 40 \%$ yield with the recovery of benzaldehyde ( $>30 \%$ yield). 
Interestingly, the yield of the reaction was still poor (below $40 \%$ ) when the reaction was performed at high temperature (at or above $40^{\circ} \mathrm{C}$ ). This is presumably due to the decomposition of the $N$-unsubstituted imine intermediate under the basic condition at high temperature. This undesired reaction of $N$-unsubstituted imine seems to be minimized under the mild reaction condition developed by us.

12 In this case, the allenyl amine was not observed, thus confirming the proposed mechanism shown in Scheme 2 .

13 (a) The diastereoselectivity was determined by the integration of ${ }^{1} \mathrm{H}$ NMR of the crude mixture; (b) The stereochemistry of the major product was confirmed by the conversion into the known allyl derivative. For detailed procedure and information on the structure determination, see the ESI. $\dagger$

14 (a) B. M. Trost and Y. H. Rhee, J. Am. Chem. Soc., 2003, 125, 7482; (b) B. M. Trost and Y. H. Rhee, J. Am. Chem. Soc., 1999, 121, 11680; (c) F. E. McDonald, K. S. Reddy and Y. Díaz, J. Am. Chem. Soc., 2000, 122, 4304.

15 L. Cui, C. Li and L. Zhang, Angew. Chem., Int. Ed., 2010, 49, 9178.

16 For our recent contribution in this area, see: $(a) \mathrm{H}$. Kim and Y. H. Rhee, J. Am. Chem. Soc., 2012, 134, 4011; (b) C. Kim, W. Lim and Y. H. Rhee, Bull. Korean Chem. Soc., 2010, 31, 1465; (c) C. Kim, H. J. Bae, J. H. Lee, W. Jeong, H. Kim, V. Sampath and Y. H. Rhee, J. Am. Chem. Soc., 2009, 131, 14660. 